\title{
An efficient approach to the cyclotrimerisation of alkynes: solvent-free synthesis of 1,3,5-trisubstituted benzenes using $p$-toluenesulfonic acid monohydrate
}

\section{Qi Gao, ${ }^{\text {a }}$ Fang-ping Bao, ${ }^{\text {a }}$ Xiao-jing Feng, ${ }^{\text {a }}$ Ying-ming Pan, ${ }^{\text {a }}$ Heng-shan Wang, and Dian-peng $\mathrm{Li}^{\mathrm{b}}$ *}

\author{
${ }^{a}$ Key Laboratory for the Chemistry and Molecular Engineering of Medicinal Resources \\ (Ministry of Education of China), School of Chemistry \& Chemical Engineering of Guangxi \\ Normal University, Guilin 541004, People's Republic of China. \\ ${ }^{b}$ Guangxi Key Laboratory of Functional Phytochemicals Research and Utilization, Guangxi \\ Institute of Botany, Chinese Academy of Sciences, Guilin 541006, People's Republic of China. \\ E-mail:panym2004@yahoo.com.cn, ldp@gxib.cn
}

\begin{abstract}
An environmentally friendly, efficient method for transforming alkynes into substituted benzenes catalyzed by $p$-toluenesulfonic acid monohydrate $\left(p-\mathrm{TsOH} \cdot \mathrm{H}_{2} \mathrm{O}\right)$ under solvent-free conditions has been developed, which conforms to the principles of "green" chemistry and overcomes the shortcomings of previous methods for the synthesis of substituted benzenes. The reaction is quite general and provides good to excellent yields.
\end{abstract}

Keywords: Alkynes, $\quad p-\mathrm{TsOH} \cdot \mathrm{H}_{2} \mathrm{O}$, cyclotrimerization, solvent-free, 1,3,5-trisubstituted benzenes

\section{Introduction}

The development of new transformations that are not only efficient, selective, and high-yielding but that are also environmentally benign is one of the challenge chemists are facing. ${ }^{1,2}$ During the last decades, the topic of "green" chemistry has received increasing attention. "- "Green" chemistry aims at the total elimination or at least the minimization of generated waste and the implementation of sustainable processes through the adoption of 12 fundamental principles. An alternative strategy to reduce the $E$-factor of reactions and their impact on the environment is to conduct them under solvent-free conditions. Solvent-free reactions have attracted considerable attention due to environmental safety, the economic viewpoint, easy work-up, high yields of the products and (usually) their short reaction times. ${ }^{5-10}$ 
The formation of benzene rings from alkynes, named the Reppe cyclotrimerization, ${ }^{11}$ has been well known as a useful method and intensively studied in the last fifty years because of the difficulty in obtaining functionalized aromatic compounds. ${ }^{12-20}$ However, most of these protocols suffer from the need of using precious metal catalysts, lower yields or harsh operating conditions, and mixtures of 1,3,5- and 1,2,4-trisubstituted benzenes are obtained in general. Recently, we reported that a novel indium(III)-catalyzed cyclotrimerization of alkynes in the presence of 2iodophenol gave 1,3,5-substituted benzenes with complete regioselectivity. However, the indium(III)-catalyzed cyclotrimerization was less successful with alkynylsilanes (only $15 \%$ yield of $\mathbf{2 a}$ ) and needed to be carried out in sealed tubes. ${ }^{21}$ Meanwhile, considering their expensive nature, inadequate accessibility, toxicity of the additives often used, the generation of toxic waste and the use of organic solvent as well as harsh conditions, there is an urgent need to develop a powerful method with a high regioselectivity to meet the requirement of green chemistry. Here we describe a green approach towards the regioselective synthesis of 1,3,5-trisubstituted benzenes via the $p$-Ts $\mathrm{OH} \cdot \mathrm{H}_{2} \mathrm{O}$-catalyzed ${ }^{22-28}$ trimerization of alkynes under solvent-free conditions.

\section{Results and Discussion}

In order to identify the optimal reaction conditions, 1-phenyl-2-trimethylsilylacetylene 1a was chosen as a model substrate. Firstly, the cyclotrimerization was carried out in the presence of 10 mol\% TfOH under solvent-free conditions and the desired product $\mathbf{2 a}$ was isolated in $53 \%$ yield (Table 1, entry 1). The reaction using TFA, CAN, $\mathrm{H}_{2} \mathrm{SO}_{4}, \mathrm{HNO}_{3}$ and $\mathrm{H}_{3} \mathrm{PO}_{4}$ as catalysts produced 2a only in low yields (Table 1, entries 2, 3 and 10-12). Changing the catalyst to $p$ $\mathrm{TsOH} \cdot \mathrm{H}_{2} \mathrm{O}$ furnished the product $\mathbf{2 a}$ in $68 \%$ yield (Table 1, entry 6 ). Furthermore, a higher yield was obtained when the amount of $p-\mathrm{TsOH} \cdot \mathrm{H}_{2} \mathrm{O}$ was increased from 0.1 equiv to 0.5 equiv or 1.0 equiv (Table 1, entries 7 and 8). Other catalysts such as oxalic acid, $\mathrm{AcOH}, \mathrm{HCl}$, or Lewis acids did not promote the cyclotrimerization reaction (Table 1, entries 4, 5, 9 and 13-16).

Encouraged by the efficiency of the reaction protocol described above, the scope of the substrate was investigated. Typical results are shown in Table 2. The aromatic alkynylsilane 1c possessing an electron-donating group at the benzene ring $\left(\mathrm{R}^{1}=4-\mathrm{MeOC}_{6} \mathrm{H}_{4}\right)$ reacted smoothly and afforded the desired product $\mathbf{2 b}$ in $95 \%$ yield (Table 2, entry 3). Substrates $\mathbf{1 i}$ and $\mathbf{1 j}$ possessing electron-withdrawing groups $\left(\mathrm{R}^{1}=4-\mathrm{FC}_{6} \mathrm{H}_{4}, 4-\mathrm{BrC}_{6} \mathrm{H}_{4}\right)$ at the benzene ring were also successfully employed in the cyclotrimerization and gave the benzene derivatives $\mathbf{2} \mathbf{f}$ and $\mathbf{2 g}$ in $78 \%$ and $82 \%$ yields, respectively (Table 2, entries 9 and 10). Significantly, the desired products could be obtained in higher yields from electron rich than from electron poor alkynylsilanes. Moreover, the desilylative cyclotrimerizations of the aromatic alkynylsilanes $\mathbf{1 e}, \mathbf{1 g}, \mathbf{1 h}$ and $\mathbf{1 l}$ $\left(\mathrm{R}^{1}=4-\mathrm{MeC}_{6} \mathrm{H}_{4}, 4-\mathrm{EtC}_{6} \mathrm{H}_{4}, 4-n-\mathrm{PrC}_{6} \mathrm{H}_{4}\right.$ and $3-\mathrm{MeC}_{6} \mathrm{H}_{4}$ ) also proceeded smoothly to afford benzene derivatives $\mathbf{2 c}, \mathbf{2 d}, \mathbf{2 e}$ and $\mathbf{2 h}$ in high yields with complete regioselectivity (Table 2, entries 5, 7, 8 and 12). However, the comparable treatment of $\mathbf{1 m}$ did not form the desired 
benzene derivative $\mathbf{2} \mathbf{i}$ (Table 2, entry 13), presumably due to the steric effect of the substituent in the ortho-position of $\mathbf{1 m}$. Cicero has also reported that 1-ethynyl-2,6-dimethoxybenzene failed to afford 1,3,5-tri(2,6-dimethoxyphenyl)benzene and/or 1,2,4-tri(2,6-dimethoxyphenyl)benzene in the presence of vanadium phthalocyanine. ${ }^{29}$ In addition, the aliphatic alkynylsilanes $\mathbf{1 n}, \mathbf{1 p}$ and internal alkyl alkyne 10 in the presence of 1.0 equiv $p-\mathrm{TsOH} \cdot \mathrm{H}_{2} \mathrm{O}$ also underwent a cyclotrimerization smoothly to give the products $\mathbf{2} \mathbf{j}, \mathbf{2} \mathbf{l}$ and $\mathbf{2 k}$ in $83 \%, 86 \%$, and $85 \%$ yields, respectively (Table 2, entries 14-16). Furthermore, the terminal alkynes 1b, 1d, 1f and 1k also gave the desired results, providing the benzene derivatives in high yields (Table 2, entries 2, 4, 6 and 11).

Table 1. Screening of catalysts for the cyclotrimerization of 1-phenyl-2-trimethylsilylacetylene ${ }^{\mathrm{a}}$

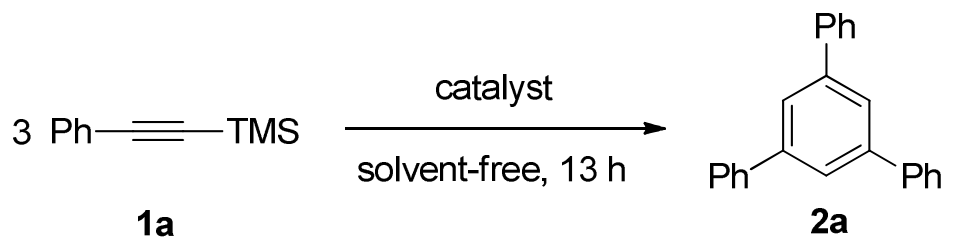

\begin{tabular}{ccc}
\hline Entry & Catalyst & Yield $(\%)^{\mathrm{b}}$ \\
\hline 1 & $\mathrm{TfOH}$ & 53 \\
2 & $\mathrm{TFA}$ & 20 \\
3 & $\mathrm{CAN}$ & 15 \\
4 & Oxalic acid & 0 \\
5 & $\mathrm{AcOH}$ & 0 \\
6 & $p$-TsOH$\cdot \mathrm{H}_{2} \mathrm{O}$ & 68 \\
7 & $p$-TsOH· $\mathrm{H}_{2} \mathrm{O}(0.5$ equiv $)$ & 79 \\
$\mathbf{8}$ & $\boldsymbol{p}$-TsOH $\cdot \mathbf{H}_{\mathbf{2}} \mathbf{O}(\mathbf{1 . 0}$ equiv $)$ & $\mathbf{9 0}$ \\
9 & $\mathrm{HCl}$ & 0 \\
10 & $\mathrm{H}_{2} \mathrm{SO}_{4}$ & 20 \\
11 & $\mathrm{HNO}_{3}$ & 28 \\
12 & $\mathrm{H}_{3} \mathrm{PO}_{4}$ & 30 \\
13 & $\mathrm{ZnCl}_{2}$ & 0 \\
14 & $\mathrm{InCl}_{3}$ & 0 \\
15 & $\mathrm{FeCl}_{3}$ & 0 \\
16 & $\mathrm{Cu}(\mathrm{OTf})_{2}$ & 0 \\
\hline
\end{tabular}

${ }^{a}$ Reaction conditions: the reactions were carried out using $\mathbf{1 a}(0.6 \mathrm{mmol})$ and catalyst $(10 \mathrm{~mol} \%)$ at $60{ }^{\circ} \mathrm{C}$ for $3 \mathrm{~h}$ and then at $140^{\circ} \mathrm{C}$ for $10 \mathrm{~h} .{ }^{b}$ Isolated yield of pure product based on $\mathbf{1 a}$. 
Table 2. Solvent-free synthesis of substituted benzenes 2 catalyzed by $p-\mathrm{TsOH} \cdot \mathrm{H}_{2} \mathrm{O}^{\mathrm{a}}$

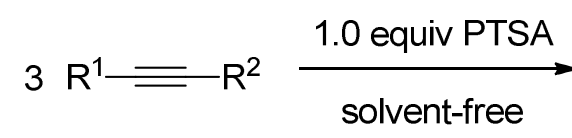

1

$\mathrm{R}^{1}=$ Aryl, Me, Et, $i-\mathrm{Pr} \quad \mathrm{R}^{2}=\mathrm{TMS}, \mathrm{H}, \mathrm{Me}$

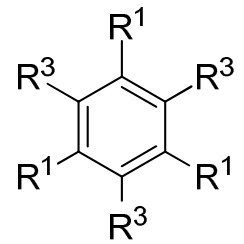

2

\begin{tabular}{|c|c|c|c|c|c|}
\hline Entry & Alkyne & \multicolumn{2}{|c|}{ Product } & Time $/ \mathrm{h}^{\mathrm{b}}$ & Yield $(\%)^{\mathrm{c}}$ \\
\hline 1 & 1a: $\mathrm{R}^{1}=\mathrm{Ph} ; \mathrm{R}^{2}=\mathrm{TMS}$ & $2 \mathbf{a}$ & $\mathrm{R}^{3}=\mathrm{H}$ & 13 & 90 \\
\hline 2 & 1b: $\mathrm{R}^{1}=\mathrm{Ph} ; \mathrm{R}^{2}=\mathrm{H}$ & $2 \mathbf{a}$ & $\mathrm{R}^{3}=\mathrm{H}$ & 14 & 87 \\
\hline 3 & $\begin{array}{c}\text { 1c: } \mathrm{R}^{1}=4-\mathrm{MeOC}_{6} \mathrm{H}_{4} \\
\mathrm{R}^{2}=\mathrm{TMS}\end{array}$ & $2 b$ & $\mathrm{R}^{3}=\mathrm{H}$ & 11 & 95 \\
\hline 4 & $\begin{array}{c}\text { 1d: } \mathrm{R}^{1}=4-\mathrm{MeOC}_{6} \mathrm{H}_{4} \\
\mathrm{R}^{2}=\mathrm{H}\end{array}$ & $2 b$ & $\mathrm{R}^{3}=\mathrm{H}$ & 13 & 94 \\
\hline 5 & 1e: $\begin{aligned} \mathrm{R}^{1} & =4-\mathrm{MeC}_{6} \mathrm{H}_{4} ; \mathrm{R}^{2} \\
& =\mathrm{TMS}\end{aligned}$ & $2 c$ & $\mathrm{R}^{3}=\mathrm{H}$ & 14 & 91 \\
\hline 6 & $\begin{array}{c}\text { 1f: } \mathrm{R}^{1}=4-\mathrm{MeC}_{6} \mathrm{H}_{4} ; \mathrm{R}^{2} \\
=\mathrm{H}\end{array}$ & $2 \mathrm{c}$ & $\mathrm{R}^{3}=\mathrm{H}$ & 15 & 89 \\
\hline 7 & $\begin{array}{c}\text { 1g: } \mathrm{R}^{1}=4-\mathrm{EtC}_{6} \mathrm{H}_{4} ; \mathrm{R}^{2}= \\
\mathrm{TMS}\end{array}$ & $2 d$ & $\mathrm{R}^{3}=\mathrm{H}$ & 15 & 88 \\
\hline 8 & 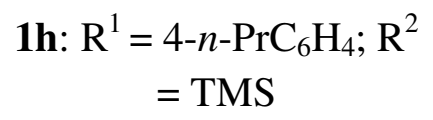 & $2 e$ & $\mathrm{R}^{3}=\mathrm{H}$ & 19 & 90 \\
\hline 9 & $\begin{array}{c}\text { 1i: } \mathrm{R}^{1}=\underset{\mathrm{TMS}}{4-\mathrm{FC}_{6} \mathrm{H}_{4} ; \mathrm{R}^{2}=} \\
\text { TMS }\end{array}$ & $2 f$ & $\mathrm{R}^{3}=\mathrm{H}$ & 24 & 78 \\
\hline 10 & $\begin{array}{c}\mathbf{1 j}: \mathrm{R}^{1}=\underset{\mathrm{TMS}}{4-\mathrm{BrC}_{6} \mathrm{H}_{4}} ; \mathrm{R}^{2}= \\
\end{array}$ & $2 \mathrm{~g}$ & $\mathrm{R}^{3}=\mathrm{H}$ & 22 & 82 \\
\hline 11 & 1k: $\mathrm{R}^{1}=4-\mathrm{BrC}_{6} \mathrm{H}_{4} ; \mathrm{R}^{2}=$ & $2 g$ & $\mathrm{R}^{3}=\mathrm{H}$ & 24 & 80 \\
\hline 12 & 11: $\mathrm{R}^{1}=3-\mathrm{MeC}_{6} \mathrm{H}_{4} ; \mathrm{R}^{2}=$ & $2 \mathrm{~h}$ & $\mathrm{R}^{3}=\mathrm{H}$ & 20 & 83 \\
\hline 13 & $\begin{array}{c}\text { 1m: } \mathrm{R}^{1}=2-\mathrm{MeOC}_{6} \mathrm{H}_{4} \\
\mathrm{R}^{2}=\mathrm{TMS}\end{array}$ & $2 \mathbf{i}$ & $\mathrm{R}^{3}=\mathrm{H}$ & 24 & 0 \\
\hline 14 & 1n: $\mathrm{R}^{1}=\mathrm{Et} ; \mathrm{R}^{2}=\mathrm{TMS}$ & $2 j$ & $\mathrm{R}^{3}=\mathrm{H}$ & 23 & 83 \\
\hline $15^{\mathrm{d}}$ & 10: $\mathrm{R}^{1}=\mathrm{Me} ; \mathrm{R}^{2}=\mathrm{Me}$ & $2 k$ & $\mathrm{R}^{3}=\mathrm{Me}$ & 21 & 85 \\
\hline
\end{tabular}


Table 2. Continued

\begin{tabular}{cccccc}
\hline Entry & Alkyne & Product & Time $^{\mathrm{b}}$ & ${\text { Yield }(\%)^{\mathrm{c}}}^{2}$ \\
\hline 16 & 1p: $\mathrm{R}^{1}=i$-Pr; $\mathrm{R}^{2}=$ TMS & 2l & $\mathrm{R}^{3}=\mathrm{H}$ & 20 & 86 \\
\hline
\end{tabular}

${ }^{\mathrm{a}}$ Reaction conditions: $0.6 \mathrm{mmol}$ of $\mathbf{1}, 0.6 \mathrm{mmol}$ of $p-\mathrm{TsOH} \cdot \mathrm{H}_{2} \mathrm{O}, 60{ }^{\circ} \mathrm{C}$ for $3 \mathrm{~h}$, then $140{ }^{\circ} \mathrm{C}$ for the rest of the time. ${ }^{\mathrm{b}}$ Reaction time used in all. ${ }^{\mathrm{c}}$ Isolated yield of pure product based on $1 .{ }^{\mathrm{d}} \mathrm{The}$ reaction was carried out in a sealed tube.
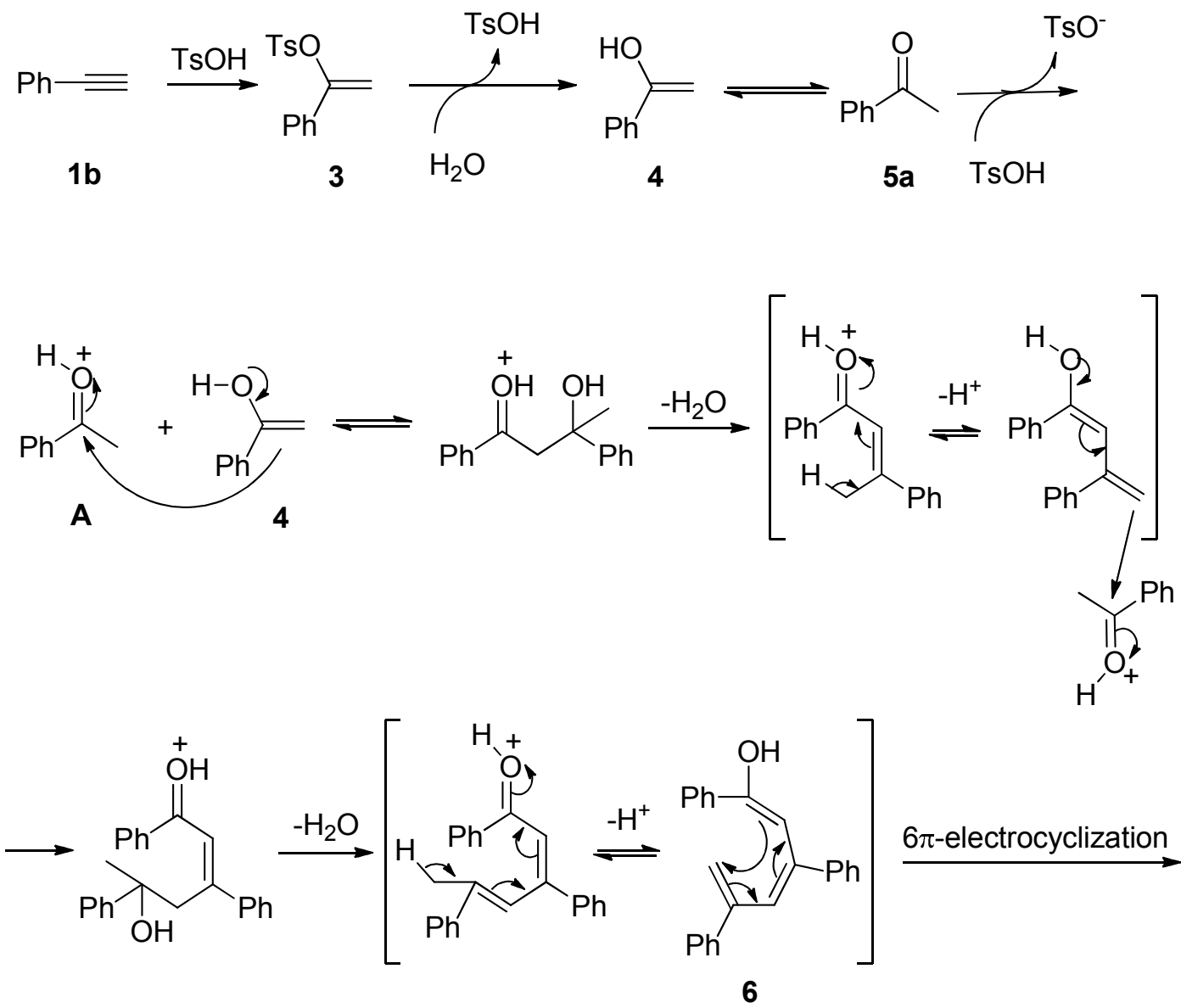<smiles>OC1(c2ccccc2)C=C(c2ccccc2)C=C(c2ccccc2)C1</smiles>

$2 a$

Scheme 1. Proposed rationale for the cyclotrimerisation of phenylacetylene. 
A possible rationale for the $p$ - $\mathrm{TsOH}$ catalyzed cyclotrimerization of arylalkynes (here exemplified for phenylacetylene 1b) is proposed in Scheme 1. Initially, $p$-TsOH attacks $\mathbf{1 b}$ to form 1-phenylethenyl tosylate, which is subsequently hydrolyzed to form the enol $\mathbf{4}$ of acetophenone $(\mathbf{5 a}){ }^{30-31}$ The latter undergoes an acid-catalyzed aldol reaction/condensation sequence to form intermediate $\mathbf{6}$, which finally, via $6 \pi$-electrocyclization followed by water elimination, yields 1,3,5-triphenylbenzene (2a). ${ }^{32}$

In order to verify our rationale, some intermediate ketones were isolated and tested under the same conditions. A satisfactory result was obtained as we had expected (Scheme 2). ${ }^{33}$

3

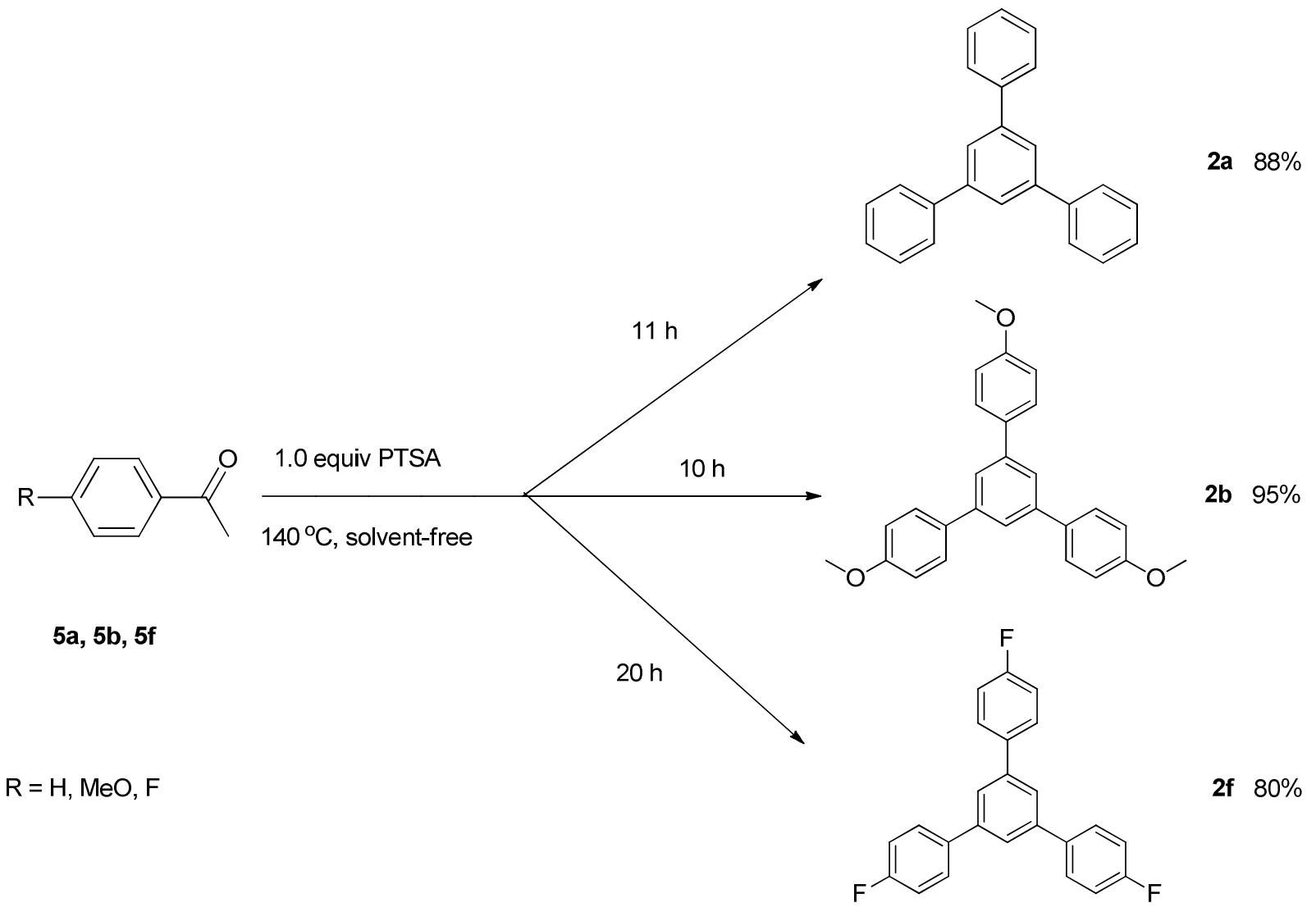

Scheme 2. Synthesis of substituted benzenes 2 from intermediate ketones 5.

\section{Conclusions}

In conclusion, we have developed an environmentally friendly, economical and efficient method for cyclotrimerization of alkynes under solvent-free conditions in the presence of $p-\mathrm{TsOH} \cdot \mathrm{H}_{2} \mathrm{O}$. The major advantages of the method lie in the cheap catalyst, easy work-up and avoidance of the 
use of toxic solvents such as DMF, DMSO, $\mathrm{CH}_{3} \mathrm{CN}$ and toluene. Air-tolerant and atomeconomical characteristics of the method accord with the concept of modern green chemistry and will be appealing for industries.

\section{Experimental Section}

General. All compounds are commercially available and were used without further purification. NMR spectra were recorded on a Bruker AVANCE DPX-400 or Bruker AVANCE DRX-500 instrument with TMS as an internal reference. MS measurements were performed on Bruker Reflex III mass spectrometer (ESI). Elemental analyses were carried out with an Elementar Vario Micro Cube in the School of Chemistry \& Chemical Engineering of Guangxi Normal University, China. Flash chromatography was performed with QingDao silica gel (300-400 mesh).

General procedure for the cyclotrimerization of alkynes 1 . To a $10 \mathrm{~mL}$ flask, alkynes $(0.6$ mmol) and $p$ - $\mathrm{TsOH} \cdot \mathrm{H}_{2} \mathrm{O}$ (1.0 equiv) were successively added. The mixture was stirred at $60{ }^{\circ} \mathrm{C}$ for $3 \mathrm{~h}$ and at $140{ }^{\circ} \mathrm{C}$ for the rest of the time, which was monitored periodically by TLC. After completion of the reaction, the reaction mixture was neutralized by saturated $\mathrm{NaHCO}_{3}$, and then extracted with $\mathrm{CH}_{2} \mathrm{Cl}_{2}(3 \times 20 \mathrm{~mL})$. The combined organic layers were dried by anhydrous $\mathrm{MgSO}_{4}$, filtered, and concentrated in vacuo. The residue was purified by flash column chromatography (PE/EA) and gave the corresponding products.

General procedure from alkynes 1 to ketones 5. To a $10 \mathrm{~mL}$ flask, alkynes $(0.6 \mathrm{mmol})$ and $p$ $\mathrm{TsOH} \cdot \mathrm{H}_{2} \mathrm{O}$ (1.0 equiv) were successively added. The mixture was stirred at $60{ }^{\circ} \mathrm{C}$ for $3 \mathrm{~h}$, which was monitored periodically by TLC. After completion of the reaction, the reaction mixture was neutralized by saturated $\mathrm{NaHCO}_{3}$, and then extracted with $\mathrm{CH}_{2} \mathrm{Cl}_{2}(3 \times 20 \mathrm{~mL})$. The combined organic layers were dried by anhydrous $\mathrm{MgSO}_{4}$, filtered, and concentrated in vacuo. The residue was purified by flash column chromatography (PE/EA) and gave the corresponding ketones $\mathbf{5}$.

1,3,5-Triphenylbenzene (2a). White solid, mp 172-174 ${ }^{\circ} \mathrm{C}$ (lit. $\left.{ }^{21,32} 170-172{ }^{\circ} \mathrm{C}\right) .{ }^{1} \mathrm{H}$ NMR $(500$ $\mathrm{MHz}_{\mathrm{CDCl}}$ ): $\delta=7.41(\mathrm{t}, J=7.2 \mathrm{~Hz}, 3 \mathrm{H}), 7.50(\mathrm{t}, J=7.3 \mathrm{~Hz}, 6 \mathrm{H}), 7.72(\mathrm{~d}, J 7.3 \mathrm{~Hz}, 6 \mathrm{H}), 7.81$ (s, 3H). ${ }^{13} \mathrm{C} \mathrm{NMR}\left(125 \mathrm{MHz}, \mathrm{CDCl}_{3}\right): \delta 125.2,127.4,127.5,128.8,141.2,142.4 . \mathrm{MS}:\left[\mathrm{M}+\mathrm{H}^{+}\right]$ 307.

1,3,5-Tris(4-methoxyphenyl)benzene (2b). White solid, mp $142-143{ }^{\circ} \mathrm{C}$ (lit. $\left.{ }^{34} 140-142{ }^{\circ} \mathrm{C}\right) .{ }^{1} \mathrm{H}$ NMR (500 MHz, $\left.\mathrm{CDCl}_{3}\right): \delta 3.88(\mathrm{~s}, 9 \mathrm{H}), 7.02(\mathrm{~d}, J 8.6 \mathrm{~Hz}, 6 \mathrm{H}), 7.63(\mathrm{~d}, J 8.6 \mathrm{~Hz}, 6 \mathrm{H}), 7.67$ (s, $3 \mathrm{H}) .{ }^{13} \mathrm{C} \mathrm{NMR}\left(\mathrm{CDCl}_{3}, 125 \mathrm{MHz}\right): \delta 55.4,114.3,123.8,128.3,133.9,141.8,159.3 . \mathrm{MS}:\left[\mathrm{M}+\mathrm{H}^{+}\right]$ 397.

1,3,5-Tris(4-methylphenyl)benzene (2c). Pale yellow solid, mp 175-176 ${ }^{\circ} \mathrm{C}$ (lit. $\left.{ }^{35} 177-178{ }^{\circ} \mathrm{C}\right)$. ${ }^{1} \mathrm{H}$ NMR (500 MHz, $\left.\mathrm{CDCl}_{3}\right): \delta 2.42(\mathrm{~s}, 9 \mathrm{H}), 7.29(\mathrm{~d}, J 7.8 \mathrm{~Hz}, 6 \mathrm{H}), 7.60(\mathrm{~d}, J 8.0 \mathrm{~Hz}, 6 \mathrm{H}), 7.73$ 
(s, 3H). ${ }^{13} \mathrm{C}$ NMR $\left(125 \mathrm{MHz}, \mathrm{CDCl}_{3}\right): \delta 21.1,124.6,127.2,129.5,137.3,138.4,142.2 . \mathrm{MS}$ :

$\left[\mathrm{M}+\mathrm{H}^{+}\right] 349$.

1,3,5-Tris(4-ethylphenyl)benzene (2d). Pale yellow solid, mp 111-113 ${ }^{\circ} \mathrm{C}$ (lit. $\left.{ }^{36} 114{ }^{\circ} \mathrm{C}\right) .{ }^{1} \mathrm{H}$ NMR (500 MHz, $\left.\mathrm{CDCl}_{3}\right): \delta 1.31(\mathrm{t}, J 7.6 \mathrm{~Hz}, 9 \mathrm{H}), 2.74(\mathrm{q}, J 7.6 \mathrm{~Hz}, 6 \mathrm{H}), 7.33(\mathrm{~d}, J 7.8 \mathrm{~Hz}, 6 \mathrm{H})$, $7.63(\mathrm{~d}, J 7.9 \mathrm{~Hz}, 6 \mathrm{H}), 7.76(\mathrm{~s}, 3 \mathrm{H}) .{ }^{13} \mathrm{C} \mathrm{NMR}\left(\mathrm{CDCl}_{3}, 125 \mathrm{MHz}\right): \delta 15.6,28.6,124.6,127.3$, 128.3, 138.7, 142.2, 143.6. MS: $\left[\mathrm{M}+\mathrm{H}^{+}\right] 391$.

1,3,5-Tris(4-n-propylphenyl)benzene (2e). Pale yellow solid, mp 141-143 ${ }^{\circ} \mathrm{C}$ (lit. ${ }^{21} 142-143$ $\left.{ }^{\circ} \mathrm{C}\right) .{ }^{1} \mathrm{H}$ NMR $\left(500 \mathrm{MHz}, \mathrm{CDCl}_{3}\right): \delta 1.00(\mathrm{t}, J 7.3 \mathrm{~Hz}, 9 \mathrm{H}), 1.75-1.67(\mathrm{~m}, 6 \mathrm{H}), 2.69-2.64(\mathrm{~m}, 6 \mathrm{H})$,

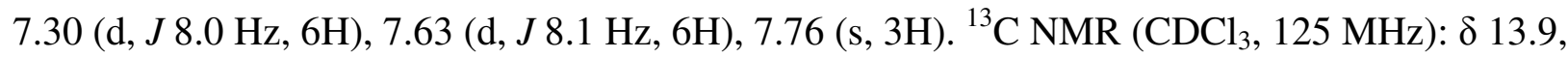
24.6, 37.7, 124.6, 127.1, 128.9, 138.6, 142.0, 142.1. MS: $\left[\mathrm{M}+\mathrm{H}^{+}\right] 433$.

1,3,5-Tris(4-fluorophenyl)benzene (2f). Yellow solid, mp 238-239 ${ }^{\circ} \mathrm{C}$ (lit. ${ }^{32} 238-240{ }^{\circ} \mathrm{C}$ ). ${ }^{1} \mathrm{H}$ NMR (500 MHz, CDCl $): \delta 7.17(\mathrm{t}, J 8.7 \mathrm{~Hz}, 6 \mathrm{H}), 7.66-7.62(\mathrm{~m}, 6 \mathrm{H}) 7.67(\mathrm{~s}, 3 \mathrm{H}) .{ }^{13} \mathrm{C}$ NMR $\left(125 \mathrm{MHz}, \mathrm{CDCl}_{3}\right): \delta 115.69$ and $115.86,124.87,128.87$ and $128.93,137.03$ and 137.05, 141.57, 161.74 and 163.70. MS: $\left[\mathrm{M}+\mathrm{H}^{+}\right] 361$.

1,3,5-Tris(4-bromophenyl)benzene (2g). Yellow solid, mp 261-263 ${ }^{\circ} \mathrm{C}$ (lit. ${ }^{32}$ 260-261 $\left.{ }^{\circ} \mathrm{C}\right) .{ }^{1} \mathrm{H}$ NMR (500 MHz, $\left.\mathrm{CDCl}_{3}\right): \delta 7.54(\mathrm{~d}, J 8.1 \mathrm{~Hz}, 6 \mathrm{H}), 7.61(\mathrm{~d}, J 8.2 \mathrm{~Hz}, 6 \mathrm{H}), 7.69(\mathrm{~s}, 3 \mathrm{H}) .{ }^{13} \mathrm{C} \mathrm{NMR}$ $\left(125 \mathrm{MHz}, \mathrm{CDCl}_{3}\right): \delta 122.1,125.0,129.0,132.1,139.7,141.6 . \mathrm{MS}:\left[\mathrm{M}+\mathrm{H}^{+}\right]$544, 542.

1,3,5-Tris(3-methylphenyl)benzene (2h). White solid, mp $117-118{ }^{\circ} \mathrm{C}$ (lit. ${ }^{37} 116.8-118.1^{\circ} \mathrm{C}$ ). ${ }^{1} \mathrm{H}$ NMR $\left(500 \mathrm{MHz}, \mathrm{CDCl}_{3}\right): \delta 2.46(\mathrm{~s}, 9 \mathrm{H}), 7.22(\mathrm{~d}, J 7.5 \mathrm{~Hz}, 3 \mathrm{H}), 7.38(\mathrm{t}, J 7.6 \mathrm{~Hz}, 3 \mathrm{H}), 7.51$ $(\mathrm{d}, J 7.6 \mathrm{~Hz}, 6 \mathrm{H}), 7.76$ (s, 3H). ${ }^{13} \mathrm{CNMR}\left(125 \mathrm{MHz}, \mathrm{CDCl}_{3}\right): \delta 21.5,124.5,125.1,128.2,128.3$, 128.7, 138.4, 141.3, 142.4. MS: $\left[\mathrm{M}+\mathrm{H}^{+}\right] 349$.

1,3,5-Triethylbenzene (2j). ${ }^{21}$ Yellow oil. ${ }^{1} \mathrm{H}$ NMR $\left(500 \mathrm{MHz}, \mathrm{CDCl}_{3}\right): \delta 1.34(\mathrm{t}, J 7.6 \mathrm{~Hz}, 9 \mathrm{H})$, 2.71 (q, J 7.6 Hz, 6H), 6.96 (s, 3H). $\left.{ }^{13} \mathrm{C} \mathrm{NMR} \mathrm{(125} \mathrm{MHz,} \mathrm{CDCl}_{3}\right): \delta 15.6,28.9,124.8,144.2$. MS: $\left[\mathrm{M}+\mathrm{H}^{+}\right] 163$.

Hexamethylbenzene (2k). White solid, mp $162-164{ }^{\circ} \mathrm{C}$ (lit. $\left.{ }^{21} 162-164{ }^{\circ} \mathrm{C}\right) .{ }^{1} \mathrm{H} \mathrm{NMR}(500 \mathrm{MHz}$, $\left.\mathrm{CDCl}_{3}\right): \delta 2.24(\mathrm{~s}, 18 \mathrm{H}) .{ }^{13} \mathrm{C} \mathrm{NMR}\left(125 \mathrm{MHz}, \mathrm{CDCl}_{3}\right): \delta 16.8,132.0 . \mathrm{MS}:\left[\mathrm{M}+\mathrm{H}^{+}\right] 163$.

1,3,5-Tri(isopropyl)benzene (2l). ${ }^{21}$ Pale yellow oil. ${ }^{1} \mathrm{H}$ NMR $\left(500 \mathrm{MHz}, \mathrm{CDCl}_{3}\right): \delta 1.36(\mathrm{~d}, J$ $7.0 \mathrm{~Hz}, 18 \mathrm{H}), 3.02-2.94$ (m, 3H), 7.01 (s, 3H). ${ }^{13} \mathrm{C} \mathrm{NMR} \mathrm{(125} \mathrm{MHz,} \mathrm{CDCl}_{3}$ ): $\delta 24.1,34.3,122.1$, 148.7. MS: $\left[\mathrm{M}+\mathrm{H}^{+}\right] 205$.

Acetophenone (5a). ${ }^{38}$ Colourless oil. ${ }^{1} \mathrm{H}$ NMR (500 MHz, $\left.\mathrm{CDCl}_{3}\right): \delta 2.58(\mathrm{~s}, 3 \mathrm{H}), 7.44-7.42(\mathrm{~m}$, 2H), 7.54-7.53 (m, 1H), 7.99-7.85 (m, 2H). $\left.{ }^{13} \mathrm{C} \mathrm{NMR} \mathrm{(125} \mathrm{MHz,} \mathrm{CDCl}_{3}\right): \delta 26.1,127.8,128.1$, 132.6, 136.6, 197.5. MS: $\left[\mathrm{M}+\mathrm{H}^{+}\right] 121$.

1-(4-Methoxyphenyl)ethanone (5b). ${ }^{38}$ Colourless oil. ${ }^{1} \mathrm{H}$ NMR $\left(500 \mathrm{MHz}, \mathrm{CDCl}_{3}\right): \delta 2.56$ (s, $3 \mathrm{H}), 3.87(\mathrm{~s}, 3 \mathrm{H}), 6.93(\mathrm{~d}, J 8.8 \mathrm{~Hz}, 2 \mathrm{H}), 7.94(\mathrm{~d}, J 8.8 \mathrm{~Hz}, 2 \mathrm{H}) \cdot{ }^{13} \mathrm{C} \mathrm{NMR}\left(125 \mathrm{MHz}, \mathrm{CDCl}_{3}\right): \delta$ 26.3, 55.4, 113.7, 130.4, 130.6, 163.5, 196.8. MS: $\left[\mathrm{M}+\mathrm{H}^{+}\right] 151$.

1-(4-Fluorophenyl)ethanone (5f). ${ }^{38}$ Colourless oil. ${ }^{1} \mathrm{H} \mathrm{NMR}\left(500 \mathrm{MHz}, \mathrm{CDCl}_{3}\right): \delta 2.58(\mathrm{~s}, 3 \mathrm{H})$, 7.16-7.09 (m, 2H), 8.02-7.94 (m, 2H). ${ }^{13} \mathrm{C} \mathrm{NMR}\left(125 \mathrm{MHz}, \mathrm{CDCl}_{3}\right): \delta 26.5,115.52$ and 115.69 , 130.86 and $130.94,133.5,164.7,166.7,196.5 . \mathrm{MS}:\left[\mathrm{M}+\mathrm{H}^{+}\right] 139$. 


\section{Acknowledgements}

This study was supported by 973 projects (2011CB512005), The projects of Key Laboratory for the Chemistry and Molecular Engineering of Medicinal Resources (Guangxi Normal University), Ministry of Education of China (CMEMR2011-15), Guangxi Natural Science Foundation of China (2012GXNSFAA053027, 2011GXNSFD018010 and 2010GXNSFF013001), and the Fund of Guangxi Key Laboratory of Functional Phytochemicals Research and Utilization (FPRU2011-1).

\section{References}

1. DeSimone, J. M. Science 2002, 297, 799.

http://dx.doi.org/10.1126/science.1069622

PMid:12161645

2. Jeon, S. J.; Li, H. M.; Walsh, P. J. J. Am. Chem. Soc. 2005, 127, 16416.

http://dx.doi.org/10.1021/ja052200m

PMid:16305227

3. Poliakoff, M.; Anastas, P. T. Nature 2001, 413, 257.

http://dx.doi.org/10.1038/35095133

PMid:11565009

4. Gawande, M. B.; Branco, P. S. Green. Chem. 2011, 13, 3355.

http://dx.doi.org/10.1039/c1gc15868f

5. Metzger, J. O. Angew. Chem. Int. Ed. 1998, 37, 2975.

http://dx.doi.org/10.1002/(SICI)1521-3773(19981116)37:21<2975::AID-

ANIE2975>3.0.CO;2-A

6. Anastas, P. T.; Warner, J. C. Green Chemistry: Theory and Practice; Oxford University

Press, Inc.: New York, 1998.

7. Sheldon, R. A. Chem. Ind. (London). 1992, 903.

8. Tanaka, K.; Toda, F. Chem. Rev. 2000, 100, 1025.

http://dx.doi.org/10.1021/cr940089p

PMid:11749257

9. Wang, D.; Li, J. H.; Li, N.; Gao, T. T.; Hou S. H.; Chen, B. H. Green. Chem. 2010, 12, 45.

http://dx.doi.org/10.1039/b917448f

10. Ando, K.; Yamada, K. Green Chem. 2011, 13, 1143. 
http://dx.doi.org/10.1039/c1gc15134g

11. Reppe, W.; Schichting, O.; Klager, K.; Toepel, T. Justus Liebigs Ann. Chem. 1948, $560,1$.

http://dx.doi.org/10.1002/jlac.19485600102

12. Zhu, Z. Y.; Wang, C. F.; Xiang, X.; Pi, C. F.; Zhou, X. G. Chem. Commun. 2006, 2066. http://dx.doi.org/10.1039/b602883g

PMid:16767277

13. Bu, X.; Zhang, Z.; Zhou, X. Organometallics 2010, 29, 3530.

http://dx.doi.org/10.1021/om100402h

14. Takeda, A.; Ohno, A.; Kadota, I.; Gevorgyan, V.; Yamamoto, Y. J. Am. Chem. Soc. 1997, 119, 4547.

http://dx.doi.org/10.1021/ja970339u

15. Lombardo, M.; Pasi, F.; Trombini, C.; Seddon, K. R.; Pitner, W. R. Green Chem. 2007, 9, 321 .

http://dx.doi.org/10.1039/b616427g

16. Tanaka, K.; Toyoda, K.; Wada, A.; Shirasaka, K.; Hirano, M. Chem.-Eur. J. 2005, 11, 1145.

http://dx.doi.org/10.1002/chem.200401017

PMid:15619723

17. Johnson, E. S.; Balaich, G. J.; Fanwick, P. E.; Rothwell, I. P. J. Am. Chem. Soc. 1997, $119,11086$.

http://dx.doi.org/10.1021/ja972306k

18. Daniel L. J, B.; Eelco, R. Synthesis 2012, 2639.

19. Czelusniak, I.; Kociecka, P.; Szymanska-Buzar, T. J. Organomet. Chem. 2012, 716, 70. http://dx.doi.org/10.1016/j.jorganchem.2012.05.044

20. Geetharani, K.; Tussupbayev, S.; Borowka, J.; Holthausen, M. C.; Ghosh, S. Chem.

Eur. J. 2012, 18, 8482.

http://dx.doi.org/10.1002/chem.201200291

PMid:22674842

21. Xu, Y. L.; Pan, Y. M.; Wu, Q.; Wang, H. S.; Liu, P. Z. J. Org. Chem. 2011, 76, 8472. http://dx.doi.org/10.1021/jo201010d; PMid:21916477

22. Augustine, J. K.; Akabote, V.; Hegde, S. G.; Alagarsamy, P. J. Org. Chem. 2009, 74, 5640 . 
http://dx.doi.org/10.1021/jo900818h

PMid:19719253

23. Chen, Y. K.; Yoshida, M.; MacMillan, D. W. C. J. Am. Chem. Soc. 2006, 128, 9328.

http://dx.doi.org/10.1021/ja063267s

PMid:16848457

24. Pan, Y. M.; Zheng, F. J.; Lin, H. X.; Zhan, Z. P. J. Org. Chem. 2009, 74, 3148.

http://dx.doi.org/10.1021/jo8027533

PMid:19354325

25. Herath, A.; Dahl, R.; Cosford, N. D. P. Org. Lett. 2010, 12, 412.

http://dx.doi.org/10.1021/o1902433a

PMid:20038130 PMCid:2814065

26. Coffinier, D.; Kaim, L. E.; Grimaud, L. Org. Lett. 2009, 11, 995.

http://dx.doi.org/10.1021/ol8029438

PMid:19170618

27. Zhu, B.; Wang, G. W. Org. Lett. 2009, 11, 4334.

http://dx.doi.org/10.1021/ol901675t

PMid:19731923

28. Peng, C. L.; Wang, Y.; Liu, L. Y.; Wang, H. G.; Zhao J. J.; Zhu, Q. Eur. J. Org. Chem. 2010, 5,818 .

http://dx.doi.org/10.1002/ejoc.200901257

29. Cicero, D.; Lembo, A.; Leoni, A.; Tagliatesta, P. New J. Chem. 2009, 33, 2162.

http://dx.doi.org/10.1039/b9nj00227h

30. Martínez, A. G.; Herrera, A.; Martínez, R.; Teso, E.; García, A.; Osío, J.; Pargada, L.; Unanue, R.; Subramanian, L. R.; Hanack, M. J. Heterocycl. Chem. 1988, 25, 1237.

http://dx.doi.org/10.1002/jhet.5570250437

31. Martínez, A.G.; Fernández, A. H.; Alvarez, R. M.; Vilar, E. T.; Fraile, A. G.; Barcina, J.O.; Iglesias, L. P. Tetrahedron Lett. 1987, 28, 1929.

http://dx.doi.org/10.1016/S0040-4039(00)96012-X

32. Kumar, A.; Dixit, M.; Singh, S. P.; Raghunandan, R.; Maulik, P. R.; Goel, A.

Tetrahedron Lett. 2009, 50, 4335.

http://dx.doi.org/10.1016/j.tetlet.2009.05.029

33. Zhao, Y.; Li, J.; Li, C.; Yin, K.; Ye, D.; Jia, X. Green. Chem. 2010, 12, 1370.

http://dx.doi.org/10.1039/c0gc00158a 
34. Kothe, G.; Zimmermann, H. Tetrahedron 1973, 29, 2305.

http://dx.doi.org/10.1016/S0040-4020(01)93354-X

35. Breschi, C.; Piparo, L.; Pertici, P.; Caporusso, A. M.; Vitulli, G. J. Organomet. Chem. 2000, 607, 57.

http://dx.doi.org/10.1016/S0022-328X(00)00211-4

36. Lyle, R. E.; DeWitt, E. J.; Nichols, N. M.; Cleland, W. J. Am. Chem. Soc. 1953, 75, 5959.

http://dx.doi.org/10.1021/ja01119a053

37. Ono, F.; Ishikura, Y.; Tada, Y.; Endo, M.; Sato, T. Synlett 2008, 15, 2365.

38. Ruan, J. W.; Li, X. M.; Saidi, O.; Xiao, J. L. J. Am. Chem. Soc. 2008, 130, 2424.

http://dx.doi.org/10.1021/ja0782955

PMid:18232688 